\title{
STABILITY AND GENERALIZED HOPF BIFURCATION THROUGH A REDUCTION PRINCIPLE
}

by

S. R. Bernfeld*

The Univeralty of Texas at Arlington

P. Negrint**

Università di Camerino, Italy

and

L. Salvador $1 *$

Università di Trento, Italy

Techntcal Report No. 140

October, 1980

UNIVE

*Thls research was partlally supported by U. S. Army Research Grant DAAG29-80-C-0060.

**Work performed under the ausplces of Itallan Councll of Research (CNR) 


\section{STABILITY AND GENERALIZED HOPF BIFURCATION}

THROUGH A REDUCTION PRINCTPLE

by

S. R. Bernfeld*, P. Negrln1**, and L. Salvador ${ }^{*}$

\section{TNTRODUCTION}

We are interested in obtaining an analysis of the bifurcating periodic orbits arlating in the generalized Hopf bifurcation problems in $R^{n}$. The existence of these periodfc otbits has often been obtained by using such techniqueg as the Liapunov-Schmidt method or topological degree arguments (Bee [5] and 1ts references). Our approach, on the other hand, is based upon stablifty properties of the equilibrium point of the unperturbed system. Andronov et. a1. [1] showed the frultfulness of this approach in studylag bifurcation problems in $R^{2}$ (for more recent papers see Negrinl and Salvador1 [6] and Bernfeld and Salvadort [2]). In the case of $R^{2}$, in contrast to that of $R^{n}, n>2$, the stability arguments can be effectively applied because of the Polncare-Bendixson theory. Blfurcation problems in $R^{n}$ can be reduced to that of $R^{2}$ when two dimensional invarlant mantfolds are known to exist. The existence of such manifolds occurs, for example when the unperturbed system contains only two purely Imaginary elgenvaluea.

In this paper we sha1l be concerned wh the general situation in $R^{n}$ in which the unperturbed gystem may have several palrs of purely

*This research was partla11y supported by U. S. Army Regearch Grant DAAG29-80-C-0060.

**Work performed under the ausplces of Itallan Counc11 of Research (CNR) 
In contrast to (a) another property which we consider in this paper is: (A) For any netghborhood $N$ of $f_{0}$, for any tnteger $l \geq 0$, for any neighborhood $U_{1}$ of 0 , and for any number $\delta>0$ thete extats $f \in N$ such that (1.2) has $f$ nontrivial pertodic orbits lylng in $U_{1}$ whose pertod is in $[2 \pi-8,2 \pi+\delta]$.

In $R^{2}$, Andronov ec.al. [1] proved that property (a) Is a consequence of the origin of (1.1) being h-asymptotically atable or it completely unatable where $h$ is an odd triteger and $k=\frac{h-1}{2}$. The origin of (1.1) in $\mathrm{R}^{\mathrm{n}}$ Is ald to be $\mathrm{h}$-asymptoclcally stable or h-completely unstable if $h$ Is the smallest positive Integer such that the origin of (1.2) Ia asymptoticalic atable (completely unstable) for al1 f for which $f(p)-f_{0}(p)=0\left(\left\|_{p}\right\|^{h}\right)$ (that is $h$ is the smallest postive integer such that agymptotic stability and complete Instabllity of the orfgin for (1.1) are recognizable by inspecting the terms up to order $h$ in the Taylor expanglon of $f_{0}$ ) (see Negrinf and Salvadorl [6] for further information on the h-asymptotlc stability). In a recent paper Bernfeld and Salvadori [2] in $R^{2}$ extended the results of Andronov et.a1. [1] by proving property (a) is equivalent to the h-asymptotie stablity (h-complete instab11ity) of the orfgin of (1.1)(where again $k=\frac{h-1}{2}$ ). It was also shown that property (A) is equivalent to the case in which the orfgin of (1.1) Is netther h-asytototically atable nor h-completely unstable for any positive integex $h$.

The problem in $\mathrm{R}^{\mathrm{n}}$ was first considered by Chafee [3]. Using the Liapunov-Schmidt method he obtained a determining equation $\psi(\xi, E)=0$ 
of a two dimensional system appropriately related to the unperturbed system (1.1). In addition, an algebralc procedure allows for a concrete solution to the problem.

In a forthcoming paper, the authors will apply an extension of the Poincaré procedure [8], glven by Salvador1 [7] In order to compute in certain cases the number $k$ directly from system (1.1).

\section{RESULTS}

By an appropriate change of coordinates depending on $f$ we may write gystems (1.1) and (1.2) respectively in the form

$$
\begin{aligned}
& \dot{x}=-y+x_{0}(x, y, z) \\
& \dot{y}=x+y_{0}(x, y, z) \\
& \dot{z}=A_{0} z+z_{0}(x, y, z),
\end{aligned}
$$

and

$$
\begin{aligned}
& \dot{x}=\alpha x-\beta y+x(x, y, z) \\
& \dot{y}=\alpha y+\beta x+Y(x, y, z) \\
& \dot{z}=A z+Z(x, y, z) .
\end{aligned}
$$

Here $\alpha, B$ are constants, $A$ and $A_{0}$ are $(n-2) \times(n-2)$ constant matrices, and $X, Y, X_{0}, Y_{0}$ belong to $C^{\infty}\left[B^{n}\left(r_{0}\right), R\right]$ and $Z, Z_{0}$ belong to $C^{\infty}\left[B^{n}\left(r_{0}\right), R^{n-2}\right]$. Moreover, $X, Y, Z, X_{0}, Y_{0}, Z_{0}$ are of order greater than one and the elgenvalues of $A_{0},\left\{\lambda_{f}\right\}_{f=1}^{n-2}$ satiafy the condition that $\lambda_{\mathrm{f}} \neq \mathrm{m}, \mathrm{m}=0, \pm 1, \ldots$. We shall refer to the right hand sides of $(2,1)$ and $(2.2)$ as $f_{0}$ and $f$ respectively. 
The two dimensional surface $z=\phi^{(h)}(x, y)$ lo tangent at the origin to the efgenspace corresponding to the efgenvalues \pm 1 . This surface will be called a quasi-invarlant mantfold of order $h$.

Given any $h>0$ define the following two dimensfonal system

$\left(s_{h}\right)$

$$
\begin{aligned}
& \dot{x}=-y+x_{0}\left(x, y, \phi^{(h)}(x, y)\right) \\
& \dot{y}=x+y_{0}\left(x, y, \phi^{(h)}(x, y)\right) .
\end{aligned}
$$

(This is the system referred to in the introduction).

We distinguish the two posaible cases:

1. There exists $h>1$ (and then $h$ muat be odd) such that $x \equiv y \equiv 0$ 1s efther h-asymptotically stable or h-completely unstable for $\left(s_{h}\right)$.

II. Case I does not hold.

We are now able to state our main reault.

Theorem 1. In case I property (a) holds with $k=\frac{h-1}{2}$. In case II, property (A) holds.

If a11 the elgenvalues of $A_{0}$ have real part not equal to zero, then for every $h>1$ there exists a $c^{h+1}$ two dimensional center manifold which w111 be denoted by $u_{h}$. We notice that if $z=\phi(x, y)$ is the equation of this center manifold, we can write

$$
\phi(x, y)=\phi^{(h)}(x, y)+o\left(x^{2}+y^{2}\right)^{h / 2} .
$$

As a corollary of Theorem 1 the following result holds.

Theorem 2. Suppose that all the elgenvalues of $A_{0}$ have real part different than zero. Then: (1) if there exists an $h$ (and $h$ must be odd) such that the origin of the unperturbed aystem (2.1) is elther h-asymptotically 
Using the transformation

$$
\zeta=z-\phi^{(h)}(x, y)
$$

we can rewrite the unperturbed system (2.1) as

$$
\begin{aligned}
& \dot{x}=-y+x_{0}^{(h)}(x, y, \zeta) \\
& \dot{y}=x+y_{0}^{(h)}(x, y, \zeta) \\
& \dot{\zeta}=A_{0} \zeta+W_{0}^{(h)}(x, y, \zeta),
\end{aligned}
$$

where $x_{0}^{(h)}(x, y, 0)=x_{0}\left(x, y, \phi^{(h)}(x, y)\right), y_{0}^{(h)}(x, y, 0)=y_{0}\left(x, y, \phi^{(h)}(x, y)\right)$. From $(2.4)$ we observe that $w_{0}^{(h)}(x, y, 0)$ is of order greater than $h$. Analogously, we can rewrite the perturbed system (2.2) as

$$
\begin{aligned}
& \dot{x}=\alpha x-B y+x^{(h)}(x, y, \zeta) \\
& \dot{y}=\alpha y+B x+y^{(h)}(x, y, \zeta) \\
& \dot{\zeta}=A \zeta+W^{(h)}(x, y, \zeta),
\end{aligned}
$$

where $X^{(h)}(x, y, 0)=X\left(x, y, \phi^{(h)}(x, y)\right), y^{(h)}(x, y, 0)=Y\left(x, y, \phi^{(h)}(x, y)\right)$ and $X^{(h)}, Y^{(h)}, W^{(h)}$ are of order $\geq 2$. For simplicity, we shall again refer to the right hand sides of $(3.1)$ and $(3.2)$ as $f_{0}$ and $f$ respectively. We now state the following lenma whose proof is based on the Implicit function theorem.

Lemma 1. There exists $L, \varepsilon, \delta>0$ and a nelghborhood $\bar{N}$ of $F_{0}$ such that for every $f \in \bar{N}$ and for every perfodic solution $\left(x\left(t, x_{0}, y_{0}, t_{0}\right)\right.$, $\left.y\left(t, x_{0}, y_{0}, \zeta_{0}\right), z\left(t, x_{0}, y_{0}, \zeta_{0}\right)\right)$ of $(3.2) 1 y$ ing in $B^{n}(\varepsilon)$ whose period 1s in 
We now Introduce for system (3.4) properties (a') and (A') which corresponds to properties (A) and (A) for system (3.2). $\left(a^{\prime}\right)(1)$ There exists a nelghborhood $N$ of $f_{0}$ and a nelghborhood $U^{\prime}$ of $r=0$, $v=0$ auch that for every $E \in N$ there are at most $k$ nontrivial $2 \pi$ perfodic solutions of (3.4) lying in $U^{\prime}$. (11) For each Integer $f, 0 \leq f \leq k$, for each neighborhood $N$ of $f_{0}$ $N \subseteq N$, and for each netghborhood $U_{1}^{\prime}$ of $r=0, v=0$ there extats $f \in N$ such that (3.4) has exactly $f$ nontrivtal $2 \pi$ partodic solutions lying in $U_{1}^{\prime}$. (A') For any neighborhood $N$ of $E_{0}$, for any integer $f \geq 0$, and for any nelghbothood $u_{1}^{\prime}$ of $r=0, v=0$ there exists $f \in N$ such that (3.4) has $f$ nontrivial $2 \pi$ periodic orbits lying in $u_{1}^{\prime}$. We then have:

Lemma 2. Property (a') Imp11es (a).

In order to prove Lemma 2 , it is sufficlent in view of Lemma 1 , to ascertain the following property: ( $b^{\prime}$ ) the $2 \pi$ pertodic solutions of (3.4) lying in a flxed nelghborhood of $r=0, v=0$ tend to the origin as $f \rightarrow f_{0}$. A solution $(r(\theta), v(\theta))$ of $(3.4)$ w111 be called a $(2 \pi, v)$ solution if $v(2 \pi)=v(0)$. Every $2 \pi$ periodic solution is a $(2 \pi, v)$ solution but the converse is not, in general, true. In order to find the $2 \pi$ perlodic solutlons, we only need to analyze the set of $(2 \pi, v)$ solutions. Under our assumptions on $A_{0}$ we can use the implicit function theorem to derive from the second equation $\operatorname{In}(3,4)$ a $c^{\infty}$ function $t(c, f), \tau(0, f)=0$ such that a solution of (3.4) passing through $\left(0, c, v_{0}\right)$, with $f-f_{0}, c$, and $v_{0}$ sufflctently sma11, is a $(2 \pi, v)$ solution if and only if $v_{0}=\tau(c, f)$. Denote 
for $\left(S_{h}\right)$ we have

$$
u_{1}\left(\theta, f_{0}\right) \equiv 1, u_{1}\left(2 \pi, f_{0}\right)=0,1=2 \ldots h-1, u_{h}\left(2 \pi, f_{0}\right) \neq 0,
$$

thus Implying (3.10) holds (see [6] for more detalls).

Let $u s$ extend the domain of $V(c, f)$ to include negative values of $c$. Since the origin 18 a solution of (3.4) for any $f$, an application of Rolle's Theorem, in view of (3.10), Implles that there exists a $\delta>0$, and a nelghborhood $N$ of $f_{0}$ such that for any $f \in N, V(c, f)$ has at most $h-1$ nonzero roots 1 ying in $[-\delta, \delta]$. On the other hand, it is easy to recognize that for each positive root of $V(c, f)$ there is a negative root of $V(c, f)$. Thus, there are at most $\frac{h-1}{2} 2 \pi$ perlodic solutions of (3.4) lying in a neighborhood $U^{\prime}$ of $r=0, v=0$. This proves (a')(1) 18 satiofted.

Property (a')(11) can be proved by assuming a particular perturbed system of the form

$$
\begin{aligned}
& \dot{x}=-y+x_{0}^{(h)}(x, y, \zeta)+\sum_{1=0}^{(h-3) / 2} a_{1} x\left(x^{2}+y^{2}\right)^{1} \\
& \dot{y}=x+Y_{0}^{(h)}(x, y, \zeta)+\sum_{1=0}^{(h-3) / 2} a_{1} y\left(x^{2}+y^{2}\right)^{1} \\
& \dot{\zeta}=A_{0} \zeta+W_{0}^{(h)}(x, y, \zeta),
\end{aligned}
$$

where $a_{1}$ are constants depending on $f, 0 \leq f \leq k, N$ and $u_{1}$.

Since the roots of $V(\cdot, f)=0$ approach zero as $E \rightarrow f_{0}$, property $\left(b^{\prime}\right)$

holds. Lemma 2 then Implies (a) holds, proving, Theorem 1 for case 1 . 


\section{BIBLIOGRAPHY}

(1) Andronov, A., Leontov1ch, E., Gordon, I., and Mater, A., Theory of Bifurcations of Dynamical Systems in the Plane, Iarae1 Program of Sclentific Translations, Jerusalem, (1971).

[2] Bernfeld, s. and Salvador1, L., Generalized Hopf Bifurcation and hAsymptotic Stability, J. Nonlinear Analysis, T.M.A. (to appear).

[3] Chafee, N., Generalized Hopf Bifurcation and Perturbation in a Full Neighborhood of a Given Vector Field, Indiana Untv. Math. J. 27 (1978), 173-194.

[4] Ltapounov, A. M., Problème Général de la Stabilité du Mouvement, Ann. of Math Studies, 17, Princeton University Press, Princeton, New Jersey, (1947).

15] Marsden, G. and McCracken, M., The Hopf Bifurcation and its Applications, Notes In Applled Math. Sc1. 19, Springer Verlag, New York, (1976).

[6] Negrin1, P. and Salvadort, L., Attractivity and Hopf Bifurcation, J. Nonlinear Analysis, T.M.A. 3 (1979), 87-100.

[7] Salvador1, L., Sulla stabilità dell Equilibrio nei Casi critici, Ann. Mat. Pura App1. (4) LXIl (1965), 1-33.

[8] Sansone, G. and Cont1, R., Nonlineat Differential Equations, Macmi11an New York, (1964). 